\title{
Perfil clínico y epidemiológico de los casos de tuberculosis atendidos en una red de salud universitaria en Santiago de Chile entre los años 2000-2010
}

\author{
ALVARO MORGADO a, RUTH KÖHNENKAMPFa, PABLO NAVARRETE2,b, \\ PATRICIA GARCÍA ${ }^{1}$, M. ELVIRA BALCELLS ${ }^{2}$
}

\section{Clinical and epidemiological profile of tuberculosis in a university hospital in Santiago, Chile}

Background: The incidence and epidemiological profile of tuberculosis (TB) has changed significantly in the recent years in Chile. Aim: To evaluate the clinical and epidemiological characteristics of TB cases diagnosed in the last decade at a university hospital in Santiago. Material and Methods: The Mycobacterium tuberculosis culture registry of the microbiology laboratory was reviewed. Medical records of patients with a positive culture registered between 2000 and 2010 were retrieved and analyzed. Results: Two hundred forty positive Mycobacterium tuberculosis cultures were identified and the medical records of 158 of these patients were accessed for analysis. The median age was 53 years (range: 3 to 89), 55.1\% were female and nearly 42\% had extra-pulmonary TB. Among known risk factors, 32.9\% of patients were older than 65 years, $4.4 \%$ were health care workers and 3.9\% immigrants. Twenty eight percent (41/145) had some type of immunosuppression at diagnosis: $11.7 \% \mathrm{HIV}$ infection and $16.6 \%$ were using immunosuppressive drugs. In this group, a previous tuberculin skin test was done in only 5 cases (12.2\%). Adverse events related to TB treatment were reported in $21.3 \%$ of cases (17/80). No cases of fulminant hepatitis or death from this cause were identified. Four of 92 patients that had a complete follow up during treatment, died. Two of these patients were receiving steroids. Conclusions: Almost one third of TB cases occurred among immunosuppressed patients and $42 \%$ were extra-pulmonary forms. The prevention of TB reactivation in this group should be strengthened.

(Rev Med Chile 2012; 140: 853-858).

Key words: Immunosuppression; Myocbacterium tuberculosis; Risk factors; Tuberculosis.

I

a infección tuberculosa a pesar de intensos esfuerzos en campañas de control, afecta aún actualmente a casi un tercio de la población mundial. A nivel global, se estima que en el año 2010 ocurrieron 9,4 millones de casos nuevos de tuberculosis (TBC) y hubo un total de 1,7 millones de muertes atribuibles a esta causa ${ }^{1}$.

En Chile gracias a las mejorías en las con- diciones de vida y a los esfuerzos del Programa Nacional de Control de la Tuberculosis (PNT), se ha reducido significativamente la incidencia de TBC en las últimas décadas, logrando alcanzarse en el año 2000 el llamado "umbral de la fase de eliminación de tuberculosis", definido como una tasa de incidencia anual para TBC menor a 20 por cien mil habitantes ${ }^{2}$. Sin embargo, desde entonces 
la velocidad de reducción de la incidencia ha sido menor a la esperada y no se logró alcanzar la fase de eliminación avanzada (tasa de incidencia menor o igual a $10 \times 100.000$ ) presupuestada para el año 2010. Esto ha sido atribuido a diversos factores y en particular a la extrema variabilidad en las tasas de incidencia de TBC según Servicios de Salud o Regiones de Chile con poblaciones que difieren en sus condiciones socioeconómicas y origen étnico.

La Red de Salud de Universidad Católica (UC) está constituida en la actualidad por dos centros hospitalarios de alta complejidad (Hospital Clínico UC y Clínica UC San Carlos de Apoquindo) con un total de 635 camas y más de 28.500 egresos anuales. Su área de atención ambulatoria incluye cinco centros médicos y 12 unidades de toma de muestra para exámenes de laboratorio distribuidas en distintas comunas de la Región Metropolitana.

El objetivo principal del presente estudio fue conocer las características clínicas y epidemiológicas de la población con diagnóstico de TBC realizado en los últimos diez años en la Red de Salud UC, buscando identificar la presencia de factores de riesgo y su importancia relativa con el fin de poder focalizar esfuerzos diagnósticos y preventivos en los grupos de mayor riesgo de desarrollar la enfermedad.

\section{Materiales y Métodos}

Se realizó un estudio descriptivo, retrospectivo. Se revisaron los registros de cultivos positivos para Mycobacterium tuberculosis del laboratorio de microbiología de la Pontificia Universidad Católica en el período que comprendido entre enero de 2000 y diciembre del año 2010. A partir de estos, se buscaron las fichas de papel y electrónicas de todos los pacientes que hubiesen sido atendidos en la Red de Salud UC. El registro de datos se realizó según una pauta previamente estandarizada. Las variables recopiladas fueron las siguientes: sexo, edad al diagnóstico, nacionalidad, residencia al momento del diagnóstico, consumo de sustancias ilícitas o recreativas, embarazo, contacto previo con personas con TBC, antecedente de TBC previa, institucionalización, inmunodepresión, infección por VIH, comorbilidad, realización de prueba con derivado proteico purificado (PPD) previo al diagnóstico, tipo de muestra para cultivo, resultados de la baciloscopia, reacciones adversas a tratamiento anti tuberculoso y mortalidad atribuible a TBC.
Para análisis se seleccionaron sólo aquellos pacientes que tuviesen registro de al menos cuatro de las variables en estudio. Para efectos del registro se consideró cada paciente como un caso único.

El diagnóstico microbiológico fue realizado como parte de la rutina clínica en el laboratorio de microbiología de la Red de Salud UC. Este corresponde a baciloscopia mediante tinción de Ziehl-Nielsen, descontaminación de las muestras con $\mathrm{N}$-acetil.cisteina y $\mathrm{NaOH}$ al $2 \%$ y siembra en cultivo sólido (Löwestein-Jensen) y en medio líquido (7H9 de Middelbrook con incubación en el sistema BACTEC MGIT 960, BD). Se verificó que existiese la confirmación de Mycobacterium tuberculosis complex por Instituto de Salud Pública como requisito para todos los casos incluidos en el estudio.

El análisis estadístico se efectuó de forma descriptiva, calculándose promedio o mediana, rangos e intervalo de confianza (IC) de 95\% o rango intercuartil (IQR). Para la evaluación de diferencias entre grupos, se utilizó el test t de Student y se consideró como significativo una diferencia con valor $\mathrm{p}<0,05$ (dos colas).

El estudio fue presentado y aprobado por el Comité de Ética de Investigación del Hospital Clínico de la Universidad Católica.

\section{Resultados}

Se obtuvo un total de 240 cultivos positivos para Mycobacterium tuberculosis en el período en estudio. De estos, se excluyeron 82 cultivos que provenían de pacientes controlados en otros centros o con fichas con información incompleta. Se seleccionaron para análisis 158 pacientes con al menos un cultivo positivo para $M$. tuberculosis y que cumplían con los requisitos mencionados. Del total de muestras, 58,2\% (92/158) correspondió a aislados pulmonares (expectoración, biopsia pulmonar, lavado bronquioalveolar o aspirado traqueobronquial) y $41,8 \%(66 / 158)$ a aislados extrapulmonares. El 5,1\% (8/158) de los pacientes presentó cultivos positivos en más de una localización en forma concomitante y en estos casos sólo se consideró el primer cultivo identificado (1 pulmonar y 7 extrapulmonares). De las muestras extrapulmonares, la localización más frecuente correspondió a linfonodos cervicales $(10,1 \%)$, luego pleural $(7,6 \%)$, orina $(5,1 \%)$, otros linfonodos $(3,8 \%)$, peritoneal $(3,2 \%)$, vertebral 
(2,5\%) y líquido cefalorraquídeo (2,5\%) (Tabla 1$)$. La sensibilidad de las baciloscopias en relación al cultivo fue de $40,2 \%$ para muestras respiratorias y de sólo 31,8\% para muestras extrapulmonares.

La mediana de edad de los pacientes fue de 53 años [rango de 3 a 89 años] y 55,1\% de sexo femenino (Figura 1). Los principales factores de riesgo para TBC presentes al diagnóstico se presentan en la Tabla 2. Del total de casos, 32,9\% correspondieron a adultos mayores de 65 años, $4,4 \%(5 / 114)$ a profesionales de la salud (médicos, arsenalera, matrona y odontólogo) y 3,9\% (6/155) a inmigrantes (Perú, Ecuador, Brasil, España e Italia). No se registraron casos en indigentes o reos, si bien estos pacientes no corresponden a la población habitualmente consultante en este centro, y tampoco se encontró registro consistente de la magnitud de consumo de alcohol de manera a poder categorizar este factor de riesgo.

En sólo 67 de los casos incluidos se dejó constancia de la existencia o ausencia de contacto en el pasado con otra persona cursando TBC, resultando en 14,9\% de los casos este antecedente positivo. Por otra parte, 19,6\% (22/112) de los pacientes refería haber tenido previamente TBC. La mediana de edad estos pacientes era de 66 años lo que es significativamente superior a la mediana del grupo total (53 años, $\mathrm{p}<0,05$ ).

Respecto a la distribución de comorbilidades, $10,1 \%$ de los casos presentaba antecedente de patología respiratoria crónica, $8,2 \%$ diabetes mellitus, 5,1\% cáncer previo o concomitante, 1,3\% insuficiencia renal crónica, $0,6 \%$ daño hepático crónico y 7,6\% más de una de las patologías an-
Tabla 1. Sitio de aislamiento de Mycobacterium tuberculosis

\begin{tabular}{|lrr|}
\hline Zona cultivo & n & \multicolumn{1}{c|}{$\%$} \\
\hline Pulmonar & 92 & 58,23 \\
\hline Linfonodos cervicales & 16 & 10,13 \\
\hline Pleural & 12 & 7,59 \\
\hline Orina & 8 & 5,06 \\
\hline Otros linfonodos & 6 & 3,80 \\
\hline Peritoneal & 5 & 3,16 \\
\hline Vertebra & 4 & 2,53 \\
LCR & 4 & 2,53 \\
\hline Otros* & 11 & 6,96 \\
\hline Total & 158 & 100,00 \\
\hline
\end{tabular}

*Otros: absceso muscular, testículo, tejido sinovial, piel, hígado, intestino, médula ósea, otros tejidos.

Tabla 2. Factores de riesgo para desarrollar TBC presentes en el grupo en estudio

\begin{tabular}{|lr|}
\hline Factor de riesgo & \multicolumn{1}{c|}{$\mathbf{n / n}$} \\
\hline Edad $>65$ años & $52 / 158(32,9 \%)$ \\
\hline TBC previa & $22 / 112(19,6 \%)$ \\
\hline Tratamiento inmunosupresor & $24 / 145(16,6 \%)$ \\
\hline Contacto previo & $10 / 67(14,9 \%)$ \\
VIH (+) & $17 / 145(11,7 \%)$ \\
\hline Profesional de la salud & $5 / 114 \quad(4,4 \%)$ \\
\hline Inmigrante & $6 / 155 \quad(3,9 \%)$ \\
\hline
\end{tabular}

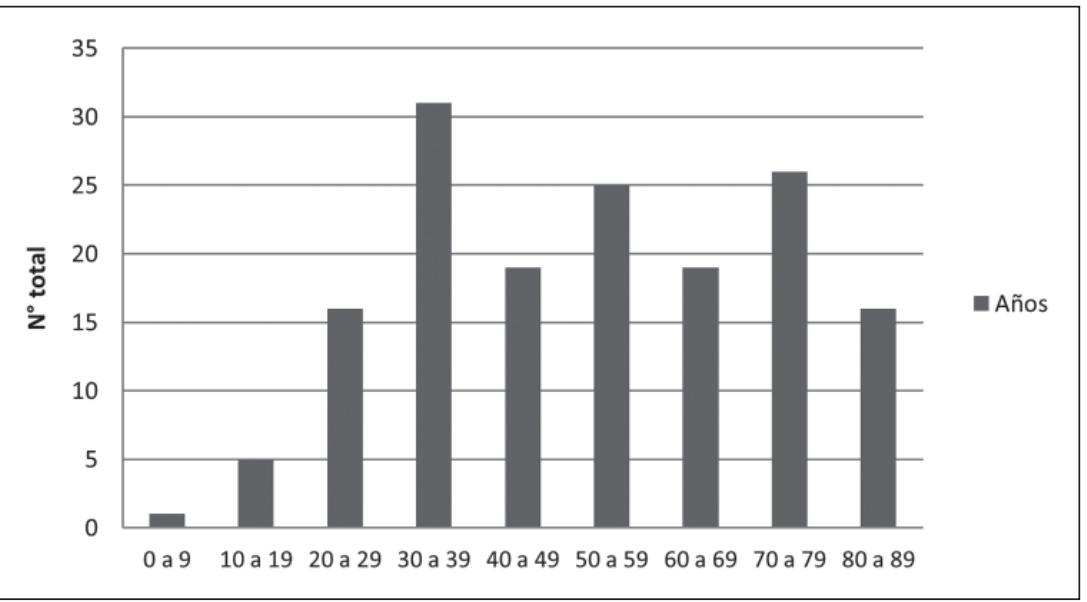

Rev Med Chile 2012; 140: 853-858
Figura 1. Distribución etaria de pacientes con tuberculosis en Hospital Clínico UC. Década 2000-2010. 
teriores simultáneamente. En $24 \%$ de los casos no se reportaba antecedentes mórbidos. Se presentó 1 caso de diagnóstico de TBC en una paciente embarazada.

El 28,3\% (41/145) de los pacientes diagnosticados con TBC presentaba algún tipo de inmunodepresión: 11,7\% asociada a infección por VIH y $16,6 \%$ secundaria a uso de drogas inmunosupresoras. De este último grupo, 50\% (12/24) correspondió a uso de corticoides sistémicos, 20,8\% (5/24) a quimioterapia por cáncer, $8,3 \%(2 / 24)$ a drogas anti-factor de necrosis tumoral (TNF) y $20,8 \%$ (5/24) a otros inmunosupresores (azatioprina, ciclofosfamida y metotrexato). La dosis diaria promedio de prednisona de aquellos pacientes bajo tratamiento esteroidal fue de $24,7 \mathrm{mg}$ (rango de 5 a $70 \mathrm{mg} /$ día) en los últimos 6 meses. Sólo en $5(12,2 \%)$ de los casos de TBC en este grupo de inmunosuprimidos existía registro de PPD previo al desarrollo de TBC y 3 resultaron positivos, todos mayores a $10 \mathrm{~mm}$.

En los pacientes con antecedente de infección por VIH predominó la TBC extrapulmonar $(59 \%$, 10/17) y la mediana de recuento de linfocitos $\mathrm{T}$ CD4 al momento del diagnóstico de TBC fue de $28,4 / \mathrm{mm}^{3}$ [IQR 27,95], con un rango entre $8,7 \mathrm{y}$ $324 \mathrm{CD} 4 / \mathrm{mm}^{3}$. En $65 \%$ de los casos el diagnóstico de TBC fue posterior al del VIH.

Con respecto a la evolución, 21,3\% (17/80) de los pacientes presentó toxicidad asociada al tratamiento con drogas antituberculosas destacando 9 casos de alteración de pruebas hepáticas, 3 casos de reacciones alérgicas, 1 caso de hiperuricemia y 1 caso de neuritis óptica. La hepatoxicidad en 8 casos fue leve [grado 1 y 2 según la escala de reacciones adversas a drogas de la Organización Mundial de la Salud (OMS)] y en 1 caso severa (grado 4$)^{3}$. No hubo casos de hepatitis fulminante o muerte por reacciones adversas en esta serie.

En los 92 casos en que se obtuvo seguimiento por ficha hasta finalizado el tratamiento, la mortalidad atribuible a TBC fue de 4,35\% (4/92). Dos de los pacientes fallecidos correspondían a pacientes usuarios de tratamiento esteroidal y los otros dos eran inmunocompetentes.

\section{Discusión}

La TBC es una enfermedad transmisible aún no erradicada en Chile por lo cual se debe reali- zar el máximo de esfuerzos en su prevención y diagnóstico precoz ${ }^{4}$. La probabilidad de progresión y reactivación de una infección tuberculosa adquirida en el pasado es mayor si esta ha sido recientemente adquirida ( $<2$ años) y en presencia de inmunodepresión ${ }^{5}$. En ambas situaciones se ha demostrado que la quimioprofilaxis con isoniazida administrada por 6 a 9 meses reduce en $60 \%$ el riesgo de progresión a TBC activa ${ }^{6,7}$.

Se han descrito múltiples factores de riesgo para el desarrollo de esta enfermedad ${ }^{8-12}$. En países desarrollados que se encuentran en vías de eliminación de la TBC, los más relevantes en la mantención de tasas altas son el aumento de la población VIH positiva y la inmigración desde países con una incidencia alta de $\mathrm{TBC}^{13}$. En nuestro país según una publicación reciente basada en registros del PNT para la Región Metropolitana (RM), 41,3\% de los casos nuevos de TBC ocurridos el año 2008 presentaba alguno de los siguientes factores de riesgo: VIH positivo (OR 29), reos (OR 15,7), situación de calle (OR $15,6)$, contacto TBC $(\mathrm{OR} 7,4)$, extranjeros (OR $2,8)$ y adultos mayores (OR 2,6$)^{14}$. Los factores de riesgo analizados en nuestra serie difieren levemente de estos, lo cual se podría explicar por incluir una población más seleccionada consultante en un hospital de nivel terciario, porque se incluyeron sólo los casos con cultivos positivos y porque se recopilaron dirigidamente factores de riesgo que habitualmente no se reportan en el formulario de enfermedades de notificación obligatoria. La proporción de coinfección TBC y VIH es levemente superior a la reportada en la publicación previamente mencionada $(11,7 \%$ vs $7,3 \%)$, pero ésta se mantiene significativamente por debajo de la prevalencia global de coinfección reportada por la OMS que en el año 2010 fue de $26 \%$ a nivel mundial ${ }^{1}$. En otro aspecto, si bien es conocido que en pacientes infectados con VIH la TBC ocurre con niveles de linfocitos T CD4 más altos que en otras infecciones oportunistas, el promedio de recuento absoluto de linfocitos T CD4 en este estudio resultó bajo y considerablemente inferior al reportado por un estudio previo de prevalencia de TBC en pacientes VIH positivos realizado en 4 diferentes regiones de Chile en el período 1998-2004 (68,8 \pm 100 versus $254 \pm 221$ cels $/ \mathrm{mm}^{3}$ ), lo cual sugiere que en el grupo actual en su mayoría pudiesen corresponder a casos de reactivación de TBC latente más que a infección 
Perfil clínico y epidemiológico de tuberculosis en hospital universitario - A. Morgado et al

de novo ${ }^{15}$. Factores como la menor prevalencia de TBC poblacional actual y la baja adherencia al uso de profilaxis tuberculosa pudiesen explicar en parte estas diferencias.

En esta serie, el uso de inmunosupresores aparece en $16,6 \%$ de los casos de TBC estudiados. Entre estas drogas, el uso de corticoides sistémicos y los agentes anti-TNF son los que se han asociado en forma más significativa y consistente en numerosos reportes a alto riesgo de reactivación de $\mathrm{TBC}^{16,17}$. Es importante, por lo tanto, buscar activamente evidencia de infección tuberculosa latente previo la administración de estos fármacos, ya sea mediante test de tuberculina cutáneo (PPD) o los nuevos test de liberación de interferón gamma (IGRA), y administrar quimioprofilaxis tuberculosa si resultan positivos. Sin embargo, los test de TBC latente deben ser interpretados con cautela si el paciente presenta inmunosupresión de tipo celular avanzada (ejemplo VIH en etapa SIDA) ya que en esta situación pueden ocurrir resultados falsos negativos, tanto para el test de PPD como para los nuevos IGRA.

Un tercio de nuestros pacientes corresponden a adultos mayores de 65 años versus al 19\% (185/957) reportado por el PNT para la $\mathrm{RM}^{14}$. El control de la TBC en pacientes mayores representa un gran desafío debido a las limitaciones existentes en el diagnóstico y en el tratamiento de la TBC latente en este grupo. En particular, se puede mencionar el claro mayor riesgo de hepatotoxicidad asociado a la quimioprofilaxis tuberculosa ${ }^{18}$.

Los trabajadores de la salud también se consideran un grupo de riesgo de desarrollar TBC por estar expuestos con mayor frecuencia a pacientes bacilíferos o a muestras respiratorias. Una revisión reciente permitió establecer que la incidencia media de TBC atribuible al trabajo en el área de la salud era de $5,8 \%$ (rango 0 a $11 \%$ ) en países de bajos y medianos ingresos y de 1,1\% (0,2-12\%) en países de altos ingresos ${ }^{19}$. En Santiago, un estudio retrospectivo de casos de TBC en el personal de salud realizado en el Servicio de Salud Metropolitano Sur reportó un riesgo cuatro a cinco veces superior al de la población general ${ }^{20}$. En nuestra serie, 4,4\% de los casos de TBC estudiados en este período de diez años correspondieron a personal de la salud. Además del adecuado cumplimiento de las normas de bioseguridad, la profilaxis efectiva y selectiva post exposición laboral y el control periódico en los sectores de riesgo debiesen ser herramientas a reforzar en los programas de salud del personal para prevenir estos casos.

En países desarrollados que tienen bajas tasas de TBC, una alta proporción de casos nuevos ocurren en inmigrantes que provienen de países con tasas elevadas. A modo de ejemplo, en Estados Unidos de Norteamérica en el año 2008, existiendo una tasa global de TBC de 4,2 casos x 100.000 habitantes, 58,5\% de los casos correspondieron a inmigrantes, lo que ha llevado a instaurar políticas de pesquisa de TBC latente y activa como requisito para ingresar a ese país ${ }^{4}$. En la serie reportada en el presente artículo, sólo 3,9\% de los pacientes correspondieron a extranjeros, lo cual puede subestimar la realidad nacional debido al espectro de población consultante a nuestro centro, ya que la proporción es menor a la reportada por el PNT (7,9\% para la RM en el año 2008) ${ }^{14}$. En Chile, si bien no existen políticas de salud dirigidas a la prevención en este grupo, se ha estimado que un aumento de la morbilidad por TBC de hasta 10-15\% atribuible a enfermos foráneos sería una situación manejable por el PNT, ya que en general y basado en la experiencia en países más desarrollados, se estima que el riesgo de contagio a partir de este grupo hacia la población general sería bajo $^{21,22}$.

Por último, en relación a la TBC secundaria a reactivación de infección antigua destaca que aproximadamente $15 \%$ de los pacientes de nuestra serie refería antecedente de contacto en el pasado con persona cursando TBC y que uno de cada 5 pacientes reportaba historia de TBC previa. En el primer caso, estos corresponderían a pacientes que tuvieron infección tuberculosa sin recibir tratamiento profiláctico y en el segundo caso probablemente traduce un tratamiento incompleto o inadecuado con persistencia de bacilos viables en estado latente o bien reinfección.

En conclusión de acuerdo a los hallazgos reportados en este estudio, se aprecia que los casos de TBC diagnosticados en la última década en este centro universitario de la RM, pertenecen en más de un tercio a grupos de pacientes vulnerables en los cuales se pudiese intervenir mediante pesquisa y tratamiento de infección tuberculosa latente. Por otra parte, la identificación de grupos en riesgo de desarrollar TBC permitiría realizar diagnóstico anticipatorio y tratamiento precoz, para así disminuir la incidencia de enfermedad activa en nuestra población. 


\section{Referencias}

1. World Health Organization. Global Tuberculosis Control: WHO report 2010. [Online] extraído de http:// www.who.int/entity/tb/publications/global_report/2010/ en/index.html [Consultado el 28 de noviembre de 2011]

2. Manual de Organización y Normas Técnicas. Programa nacional de Control de la Tuberculosis 2005. Subsecretaría de Salud Pública, División de Prevención y Control de Enfermedades.

3. World Health Organization. International Monitoring of Adverse Reactions to Drugs: Adverse Reaction Terminology. Uppsala: WHO Collaborating Center for International Drug Monitoring, 1992.

4. Tabilo F. La búsqueda de nuevos tuberculosos: Prevención y acción en la comunidad y Hospitales. Rev Chil Enf Respir 2008; 24: 317-22.

5. Jasmer RM, Nahid P, Hopewell P. Latent tuberculosis infection. N Engl J Med 2002; 347: 1860-6.

6. Woldehanna S, Volimink J. Treatment of latent tuberculosis infection in HIV infected persons. The Cochrane Database of Systematic Reviews 2004, Issue 1.

7. Smieja MJ, Marchetti CA, Cook DJ, Smaill FM. Isoniazid for Preventing Tuberculosis in Non-HIV Infected Persons (Cochrane Review). The Cochrane Library, Issue 2. Chichester, UK: John Wiley \& Sons, 2004.8) Nava-Aguilera E, Anderson N, Harris E, Mitchell S, Hamel C, Shea $\mathrm{B}$ et al. Risk factors associated with recent transmission of tuberculosis: systematic review and meta-analysis. Int J Tuberc Lung Dis 2009; 13 (1): 17-26.

9. Jeon CY, Murray MB. Diabetes mellitus increases the risk of active tuberculosis: a systematic review of 13 observational studies. PLoS Med 2008; 5 (7): e152.

10. Lönnroth K, Williams BG, Stadlin S, Jaramillo E, Dye C. Alcohol use as a risk factor for tuberculosis - a systematic review. BMC Public Health 2008; 8: 289.

11. Fok A, Numata Y, Schulzer M, Fitz Gerald MJ. Risk factors for clustering of tuberculosis cases: a systematic review of population-based molecular epidemiology studies. Int J Tuberc Lung Dis 2008; 12 (5): 480-92.

12. Slama K, Chiang CY, Enarson DA, Hassmiller K, Fanning A, Gupta P, et al. Tobacco and tuberculosis: a qualitative systematic review and meta-analysis. Int J Tuberc Lung Dis 2007; 11 (10): 1049-61.

13. Center for Diseases Control. Trends in Tuberculosis Incidence. United States, 2008. MMWR 2009; 58 (10): 249-53.

14. García C. Tuberculosis en grupos de riesgo en la Región Metropolitana. 2008. Rev Chil Enf Respir 2010; 26: 10511.

15. Villarroel L, Rabagliati R, Balcells ME, Karzulovic L, Pérez C. Tuberculosis en individuos con infección por VIH en Chile: Estudio de prevalencia e impacto sobre mortalidad. Rev Med Chile 2008; 136 (5): 578-86.

16. Brassard P, Kezouh A, Suissa S. Antirheumatic drugs and the risk of tuberculosis. Clin Infect Dis 2006; 43: 717-22.

17. Kharbanda P, Dagaonkar R, Balakrishnan C, Udwadia ZF. Tumor necrosis factor-alpha blocker induced tuberculosis. J Rheumatol 2010; 37 (7): 1542.

18. Mori T, Leung CC. Tuberculosis in the global aging population. Infect Dis Clin North Am 2010; 24 (3): 751-68.

19. Menzies D, Joshi R, Pai M. Risk of tuberculosis infection and disease associated with work in health care settings. Int J Tuberc Lung Dis 2007; 11 (6): 593-605.

20. Fica A, Ramonda P, Jemenao MI, Zambrano A, Cifuentes M, Febré N, et al. Tuberculosis en el personal de salud del Servicio Metropolitano Sur de Santiago, Chile. Rev Chil Infect 2009; 26 (1): 34-8.

21. Yáñez del A. Tuberculosis en inmigrantes. Situación Chile-Perú. Rev Chil Enf Respir 2010; 26: 161-4.

22. Das D, Baker M, Venugopal K, McAllister S. Why the tuberculosis incidence rate is not falling in New Zealand. NZMJ 2006; 119 (1243): U2248. 